\title{
ICAM-1 depletion in the center of immunological synapses is important for calcium releasing in T-cells
}

\author{
Yuanzhen Suo*,**, Wei Lin ${ }^{\dagger}+* *$, Yuting Deng ${ }^{\dagger}$, Zhichao Fan*, Lizeng Qin*, \\ Guosheng Jiang*, Yiwei Chu ${ }^{\dagger, \pi, \|}$ and Xunbin $\mathrm{Wei}^{*, \S, \|}$ \\ *Med-X Research Institute and School of Biomedical Engineering \\ Shanghai Jiao Tong University, Shanghai 200030, P.R. China \\ ${ }^{\dagger}$ Department of Immunology and Key Laboratory of Medical Molecular \\ Virology of $\mathrm{MOE} / \mathrm{MOH}$ \\ School of Basic Medical Sciences, and Biotherapy Research Centre \\ Fudan University, Shanghai 200032, P.R. China \\ ${ }^{\star}$ Shandong Academy of Medical Sciences, Jinan 250030, P.R. China \\ \$xwei01@sjtu.edu.cn \\ 『ywchu@shmu.edu.cn
}

Received 17 September 2016

Accepted 11 April 2017

Published 22 June 2017

\begin{abstract}
T-cell activation requires the formation of the immunological synapse (IS) between a T-cell and an antigen-presenting cell (APC) to control the development of the adaptive immune response. However, calcium release, an initial signal of T-cell activation, has been found to occur before IS formation. The mechanism for triggering the calcium signaling and relationship between calcium release and IS formation remains unclear. Herein, using live-cell imaging, we found that intercellular adhesion molecule 1 (ICAM-1), an essential molecule for IS formation, accumulated and then was depleted at the center of the synapse before complete IS formation. During the process of ICAM-1 depletion, calcium was released. If ICAM-1 failed to be depleted from the center of the synapse, the sustained calcium signaling could not be induced. Moreover, depletion of ICAM-1 in ISs preferentially occurred with the contact of antigen-specific T-cells and dendritic cells (DCs). Blocking the binding of ICAM-1 and lymphocyte function-associated antigen 1 (LFA-1), ICAM-1 failed to deplete at the center of the synapse, and calcium release in T-cells decreased. In studying the mechanism of how the depletion of ICAM-1 could influence calcium release in T-cells, we found that the movement of ICAM-1 was associated with the localization of LFA-1 in the IS, which affected the localization of calcium microdomains, ORAI1 and mitochondria in IS. Therefore, the depletion of ICAM-1 in the center of the synapse is an important factor for an initial sustained calcium release in T-cells.
\end{abstract}

Keywords: T-cell activation; immunological synapse; ICAM-1; calcium signaling.

$\|_{\text {Corresponding authors. }}$

${ }^{* *}$ These two authors contributed equally to this study.

This is an Open Access article published by World Scientific Publishing Company. It is distributed under the terms of the Creative Commons Attribution 4.0 (CC-BY) License. Further distribution of this work is permitted, provided the original work is properly cited. 


\section{Introduction}

The formation of the immunological synapse (IS) is a dynamic process in which a multi-molecular assembly of receptors and adhesion molecules accumulates at the interface of T-cell and antigenpresenting cells (APCs). It plays a vital role in controlling T-cell activation. ${ }^{1}$ When a T-cell recognizes the artificial planar bilayer anchored with major histocompatibility complex-peptide (MHC-peptide) and intercellular adhesion molecule 1(ICAM-1), ${ }^{1}$ a broad central zone of ICAM-1 accumulation is firstly established in the IS. At the same time, the T-cell receptor-peptide-MHC (TCRpMHC) is detected in the outer region of the IS. Then, ICAM-1 moves from the center to the periphery of the IS to form a ring around the TCRpMHC. ${ }^{1}$ This classical matured IS is called a bullseye IS. ${ }^{1,2}$ Another type of matured IS is the multifocal IS, which is characterized by interspersed ICAM-1 molecules among multiple small accumulations of TCR-pMHC complexes and phosphorylated signaling molecules at the T-DC interface. ${ }^{3,4}$ The process of both types of synapses is characterized by ICAM-1 accumulation and exclusion from the center to the periphery of the synapse. Moreover, both mature synapses are believed to be correlated with T-cell activation. However, the mechanism of how synapses control T-cell activation is still unknown.

Calcium release is an initial marker for T-cell activation, and facilitates the organization of the IS. Calcium release supports the redistribution of receptors, signaling molecules and organelles toward the T-cell-APC interface to induce downstream signaling events and ultimately support Tcell functions. ${ }^{5,6}$ However, the mechanism of how to trigger the calcium signaling remains unclear. Previous reports have shown that the calcium response could be initiated after a few seconds of interaction of T-cells and DCs, before the IS was completely formed. ${ }^{1,5-9}$ This early calcium response requires the accumulation of a small amount of adhesion molecules and a few TCR-pMHC pairs. It was estimated that a few tens of specific TCRpMHC pairs at the surface of one DC were sufficient to trigger such a signal. ${ }^{8}$ Even a single TCRpMHC might be sufficient to trigger a small and transient calcium response. ${ }^{10}$ However, Revy et al. ${ }^{11}$ showed that in T-cell-DC synapses formed in the absence of antigens and MHC molecules, ICAM-1, LFA-1 and CD43 accumulation were involved and led to a number of T-cell responses: a local increase in tyrosine phosphorylation, small calcium responses, weak proliferation and long-term survival. These responses indicated that adhesion-molecule accumulation at synapses was able to initiate calcium release. However, the mechanism of how adhesion molecules participate in calcium release is unclear.

ICAM-1 is an essential molecule in IS formation and T-cell activation and is dynamically transported from the center to the periphery of the synapse. ${ }^{1,2,12}$ Recently, ICAM-1 clustering has been proven to promote ligand-dependent LFA-1 activation in T-cells to contribute to T-cell priming and adhesion. ${ }^{13}$ Conversely, constraining ICAM-1 mobility opposes the force on LFA-1 exerted by the Tcell cytoskeleton. ${ }^{13}$ Thus, the dynamic mobility of ICAM-1 in the process of IS formation may affect Tcell activation by regulating LFA-1 movement. Moreover, the binding of ICAM-1 and LFA-1 could affect the migration of neutrophils and F-actin polymerization, ${ }^{14-16}$ which control the location of calcium microdomains at the IS to lead to calcium release. ${ }^{5,17}$ Therefore, the movement of ICAM-1 during IS formation may regulate calcium release in T-cells by binding with LFA-1 and regulating its movement. In our previous work, we established a DC2.4-ICAM-1-EGFP cell line and validated use of this cell line in studying the synapse formation between T-cells and DCs in the natural state of primary CD4 ${ }^{+}$T-cell immune response. ${ }^{18}$ The cell line of DCs, DC2.4, has the characteristics of DCs and can mimic their function. ${ }^{19,20}$ Here, using this model, we studied the relationship between the mobility of ICAM-1 and calcium release in T-cells, as well as the mechanism of how the mobility of ICAM-1 led to calcium release. We found out that calcium released in T-cells was correlated with ICAM-1 depletion in the center of the T-DC interface. The depletion of ICAM-1 was associated with the movement of LFA-1 that could control the location of ORAI1 and mitochondria at the IS. This finding suggested that ICAM-1 depletion in the center of immunological synapses was important for calcium release in T-cells. 


\section{Materials and Methods}

\subsection{Animals and cell culture}

OT-II transgenic mice (C57BL/6 background) were purchased from the Jackson Laboratory (Sacramento, CA, USA). C57BL/6 mice were purchased from Shanghai Slac Laboratory Animal Co., Ltd (Shanghai, China). All animals were maintained in a specific pathogen-free facility. All animal experiments were approved by the Ethics Committee of Fudan University (20120302-023) and were undertaken in accordance with the Guidelines for the Care and Use of Laboratory Animals (No. 55 issued by Ministry of Health, Peoples Republic of China on 25th January, 1998).

Primary $\mathrm{CD}^{+}$T-cells were obtained from spleens of 6-8 weeks C57BL/6 mice. OT-II CD4 ${ }^{+}$ T-cells were selected from spleens of $6-8$ weeks OTII $\times$ Rag-/- mouse by using a CD4 negative-selection kit (Miltenyi Biotech, Auburn, CA, USA). SEBspecific T-cells were positively selected by $\mathrm{PE}$ anti$\operatorname{TCRv} \beta 3,-7$ and -8$)$ antibody and anti-PEmicrobeads (Miltenyi Biotech, USA). Nonspecific antigen T-cells were obtained from CD4 ${ }^{+}$T-cells of C57BL/6 mice by removing the SEB or OVA-specific T-cells via microbeads with the anti-specific TCR antibody. Cells were cultured in RPMI 1640 medium (Sigma, St. Louis, MO, USA) containing $10 \%$ FBS (Atlanta Biologicals, USA) and supplemented with $1 \mathrm{mM}$ L-glutamine, $50 \mu \mathrm{M}$ 2-mercaptoethanol (2-ME), and $1 \mathrm{mM}$ nonessential amino acids at $37^{\circ} \mathrm{C}$ in a $5 \% \mathrm{CO}_{2}$ incubator. The $\mathrm{DC} 2.4$ cell line was a kind gift from Professor Kenneth L. Rock (University of Massachusetts Medical School) and cultured in RPMI 1640 containing 10\% FBS at $37^{\circ} \mathrm{C}$ in a $5 \% \mathrm{CO}_{2}$ incubator. The ICAM-1EGFP/DC2.4 cell line was established and cultured as previously reported. ${ }^{18}$

\subsection{Reagents and antibodies}

The peptide $\mathrm{OVA}_{(323-339)}$ and recombinant murine interferon gamma (IFN- $\gamma$ ) were purchased from Sigma-Aldrich (St Louis, MO, USA) and PeproTech (Rocky Hill, NJ, USA), respectively. For live cell imaging, H57-597-Fab-TCR $\alpha \beta$-Alexa Fluor 647 (Molecular Probes, Carlsbad, CA, USA) and H155-F(ab)-LFA-1(CD11a/CD18)-PE (Biolegend, San Diego, CA, USA) were used as nonblocking antibodies. $^{21,22}$ For calcium imaging, Calcium Crimson $^{\mathrm{TM}}$ (Molecular Probes, Carlsbad, CA,
USA) was used. For LFA-1 blocking, anti-LFA-1 antibody (anti-CD11a and anti-CD18) was used to pretreat T-cells before contacting DCs. For ICAM-1 blocking, anti-ICAM-1 antibody $(10 \mu \mathrm{g} / \mathrm{mL})$ was used to pretreat DCs $\left(2 \times 10^{6}\right)$ before contacting T cells. For ICAM-1 knockdown, siRNA duplexes against the fragment of the ICAM- 1 gene (5'CCTCCGGACTTTCGATCTT- $3^{\prime}$ ) and nontargeting siRNA control \#1 were purchased from RiboBio Co. Ltd. (Guangzhou, China). Both siRNAs were used for transfection into the DC2.4ICAM-1-GFP cell line separately. After $48 \mathrm{~h}$ of transfection, cells were used in live-cell imaging experiments, and protein knockdown was assessed by Western blotting (Supplementary Fig. 1).

\subsection{DC maturation and $\mathrm{CD}_{4}^{+}$ T-cell-DC contact}

For maturation of ICAM-1-EGFP/DC2.4 cells, IFN- $\gamma(20 \mathrm{ng} / \mathrm{mL})$ was incubated in the medium overnight. Lipopolysaccharide ((LPS), $100 \mathrm{ng} / \mathrm{mL}$ ) was added for a $1 \mathrm{~h}$ incubation. ${ }^{19,20}$ OVA $_{(323-339)}$ $(5 \mu \mathrm{M})$ and SEB $(100 \mathrm{ng} / \mathrm{ml})$ were used to pulse the DC cell line for $2 \mathrm{~h}$ and $30 \mathrm{~min}$, respectively. For live cell imaging, $\mathrm{H} 57-\mathrm{F}(\mathrm{ab})-\mathrm{TCR} \alpha \beta$-Alexa Fluor 647 was used to stain T-cells at $4{ }^{\circ} \mathrm{C}$ for $30 \mathrm{~min}$. Then, $\mathrm{T}$ cells were incubated with antigen-pulsed DCs $(\mathrm{T}: \mathrm{DC}=10: 1$ ) for imaging. Confocal microscopy was performed for live cell imaging as previously reported. ${ }^{18,23}$

\subsection{Confocal microscopy}

Images were taken with a confocal microscope (Leica TCS SP5, Leica Microsystems GmbH, Wetzlar, Germany) equipped with an APO oil immersion objective lens $(63 \times, \mathrm{NA}=1.40)$. To quantify redistribution of molecules at the contact site, T-DC doublets were selected from bright-field images and evaluated by fluorescent image stacks. Each image stack consisted of 6-15 sections with $1 \mu \mathrm{m}$ per step at the $z$-axis. Time-lapse scanning was performed for live cell imaging for $10 \mathrm{~min}$ at $10 \mathrm{~s}$ intervals with $512 \times 512$ pixels per frame.

\subsection{Calcium imaging}

For calcium imaging, OT-II CD4 ${ }^{+}$T-cells were incubated with H57-Fab-TCR $\alpha \beta$-Alexa Fluor 647 at 
$4^{\circ} \mathrm{C}$ for $30 \mathrm{~min}$, washed twice, and then labeled with $10 \mu \mathrm{M}$ Calcium Crimson ${ }^{\mathrm{TM}}$ in $1 \mathrm{~mL}$ calcium-free PBS at $25^{\circ} \mathrm{C}$ for $60 \mathrm{~min}$. Then, cells were washed twice and added to the antigen-pulsed ICAM-1EGFP/DC2.4. Afterwards, the calcium imaging in T-cells was detected as previously reported. ${ }^{18,23}$

\subsection{Image analysis}

The images were analyzed with Leica Application Suite Advanced Fluorescence software (Leica Microsystems GmbH, Germany) and Imaris software (Bitplane, Switzerland). Segmentation, rendering, area and maximum-intensity projection were used. Utilizing these tools, 3D intercellular contacts could be visualized from multiple angles. Only T-DC pairs whose contact orientation was proper for $x-y$ plane projection were taken into consideration for further analysis.

Kymographic analysis was performed essentially as described previously. ${ }^{24} \mathrm{~A}$ kymograph is created from a multi-time point image sequence and is able to display time dependent events. In brief, videos of IS formation between a DC and a T-cell and calcium response in the T-cell were collected and analyzed with ImageJ (National Institutes of Health, USA). For analysis of ICAM-1-GFP mobility in the IS, a line along the diameter of the IS was used to generate a kymographic image. For analysis of the calcium release in the T-cell, a line along the diameter of the T-cell was used to generate a kymographic image. Flow rate of ICAM-1-GFP was analyzed by Imaris software (Bitplane, Switzerland).

\subsection{Data analysis}

For all statistics, two-tailed Student's $t$-test and one-way ANOVA were applied to compare two normal distribution datasets. Mann-Whitney $U$ test was used to compare two nonparametric datasets. Significance levels and symbols employed were $\left.p<0.05\left(^{*}\right), p<0.01{ }^{* *}\right)$ and $p<0.001\left(^{* * *}\right)$.

\section{Results}

\subsection{Calcium release in $T$-cells during ICAM-1 depletion in the center of T-DC interface to form ISs}

It is well known that ICAM-1 promotes T-cell activation as a co-stimulator, but the effect of mobility of ICAM-1 on T-cell activation is incompletely understood. In previous study, ${ }^{18}$ we established a DC2.4-ICAM-1-EGFP cell model to study the IS formation between T-cells and DCs. Based on this model, here, we dynamically imaged the contact of T-cells and DC2.4-ICAM-1-EGFP cells to analyze the relationship between the movement of ICAM-1 and calcium release in T-cells. We found out that within the first 5 min of the formation of a bullseye IS, ICAM-1 accumulated at the center of the T-DC contact area, while TCR clusters moved to the interface of ICAM-1 clusters. Then, ICAM-1 began to be depleted at the center of the synapse (Figs. 1(a) and 1(b), at 200 s), followed by transportation of ICAM-1 from the center to the periphery (Figs. 1(a) and 1(b), from $200 \mathrm{~s}$ to $480 \mathrm{~s}$ ). The diameter of the depletion area of ICAM-1 increased from $0.35 \mu \mathrm{m}$ to $1.42 \mu \mathrm{m}$. Calcium in the Tcell was initially released at $280 \mathrm{~s}$ (Figs. 1(a) and 1(b)). After calcium release, there was little change in the diameter of the depletion area of ICAM-1 (Figs. 1(a) and 1(b)). There was a sustained and high level of calcium released in the T-cell at $480 \mathrm{~s}$. Meanwhile, ICAM-1 was completely depleted, while TCR clusters accumulated at the center of the synapse, indicating the mature synapse was formed (Figs. 1(a) and 1(b)). With analysis of the fluorescent distribution of ICAM-1 along the diameter of the synapse at different time points, we found out that the fluorescent value of ICAM-1 decreased in the middle of the synapse, while it increased at periphery of the synapse at $200 \mathrm{~s}$. At $480 \mathrm{~s}$, the fluorescent value of ICAM-1 was higher at the periphery than that at the center of the synapse. Meanwhile, the fluorescent value of TCR increased at the center of the synapse (Fig. 1(c)). Kymograph analysis showed the extension of ICAM-1 from center to periphery (white arrow) occurred before calcium release (white arrow) (Fig. 1(d)). During ICAM-1 depletion at the center of the synapse, the flow rate of ICAM-1 in the IS increased and then deceased slowly after the sustained calcium release in the T-cell (Fig. 1(e)).

Similar process occurred in multifocal IS formation. ICAM-1 began to be depleted at the center of a specific plane of the multifocal IS at $160 \mathrm{~s}$, while the diameter of the depletion area of ICAM-1 increased. After that, calcium signaling in the T-cell was released (Supplementary Fig. 2). We repeated the experiments in $25 \mathrm{~T}$-cells and obtained similar results. The mean time of ICAM-1 depletion before 
calcium release was $165 \pm 81 \mathrm{~s}$. The process of ICAM-1 accumulation and depletion occurred before the calcium release and complete IS formation. These results indicated that the mobility of ICAM-1 might affect calcium release and IS formation.

However, a portion of immature ISs, which only accumulated ICAM-1 within the contact between T-cells and DCs, could not induce sustained calcium signaling in T-cells (Fig. 2(a)). ICAM-1 depletion

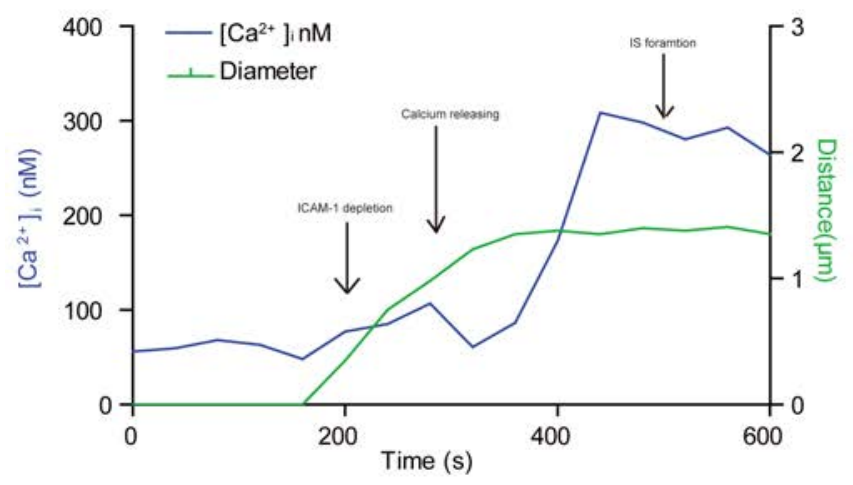

(a)
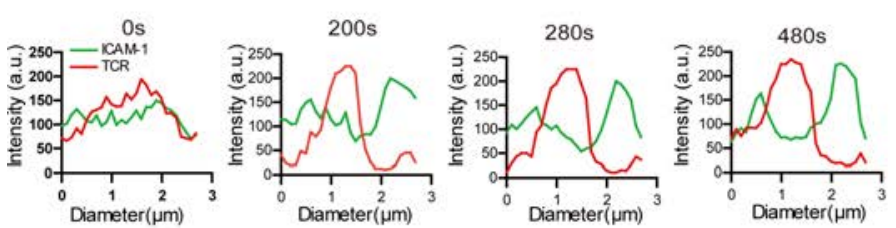

(c)

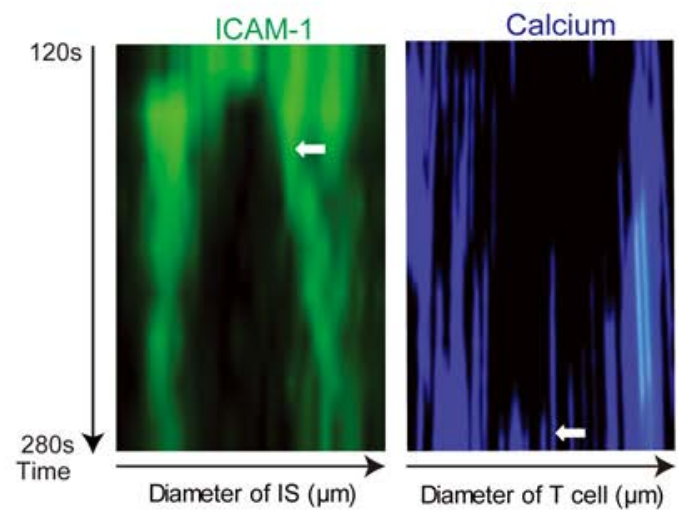

(d) did not occur in these synapses. The distribution of TCR and ICAM-1 was diffused (Fig. 2(b)). Transient and slight calcium signaling was observed in T-cells without sustained and high level of calcium signaling, which was believed to be the symbol of full T-cell activation. Analysis of the distribution of the fluorescent value of ICAM-1 in the synapse showed that the fluorescent value did not change significantly (Fig. 2(c)). Kymograph analysis could

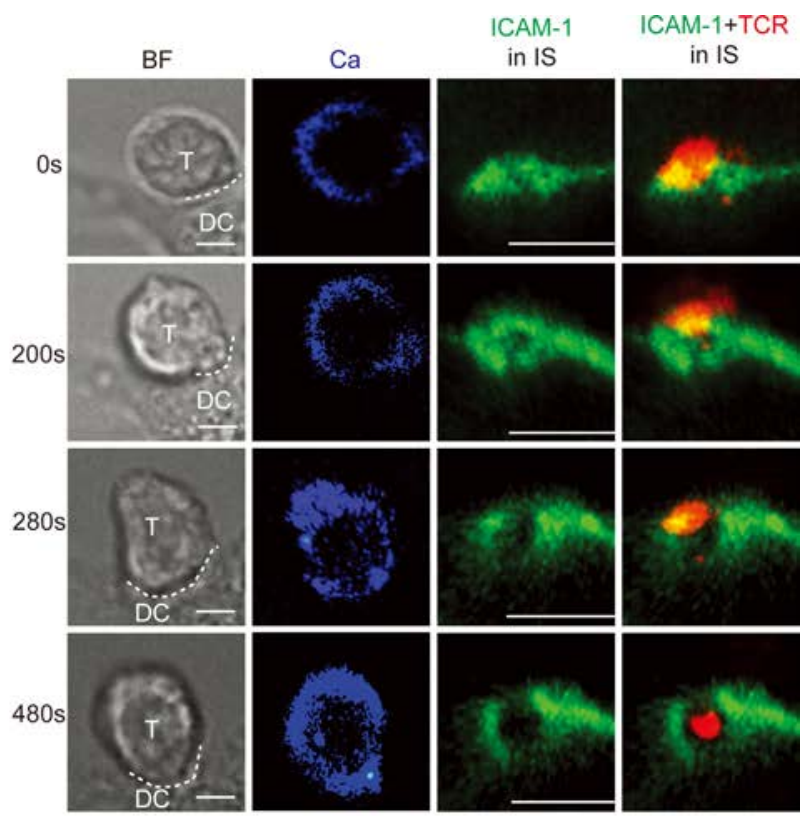

(b)

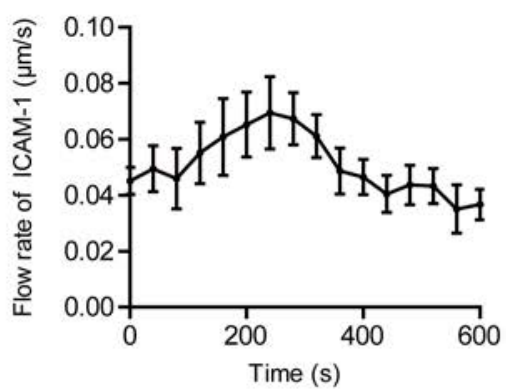

(e)

Fig. 1. ICAM-1 depletion in a bullseye IS occurred before calcium release in a T-cell: (a) The relationship between the diameter change of the area of ICAM-1 depletion and calcium signaling in a T-cell. (b) Images of calcium signaling in a T-cell and synapse formation at different time points. Bar $=2 \mu \mathrm{m}$. (c) Analysis of fluorescent distribution of ICAM-1 and TCR along the diameter of the synapse at different time points. (d) Kymograph analysis of the relationship between ICAM-1 depletion from the center to the periphery and calcium release in a T-cell. The white arrow in the left panel indicates the trend of ICAM-1 movement from the center to the periphery of the IS. The white arrow in the right panel indicates the calcium release in a T-cell. (e) The flow rates of ICAM-1 in the synapse during bullseye IS formation. 


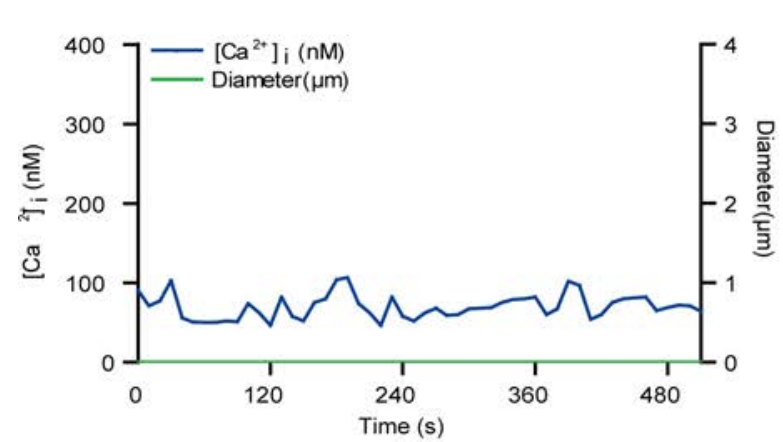

(a)

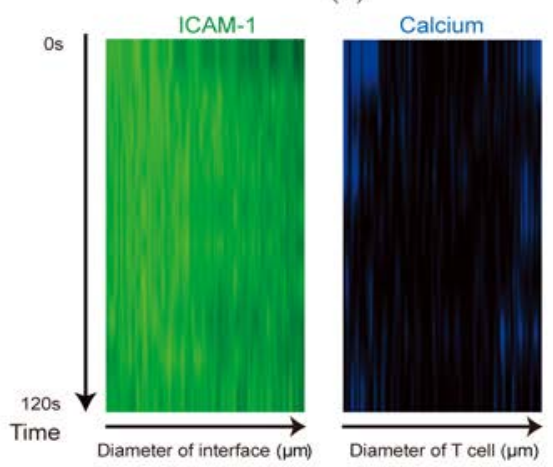

(d)
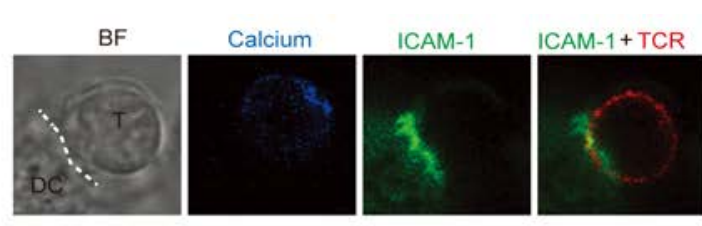

ICAM-1+TCR at interface

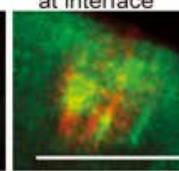

(b)
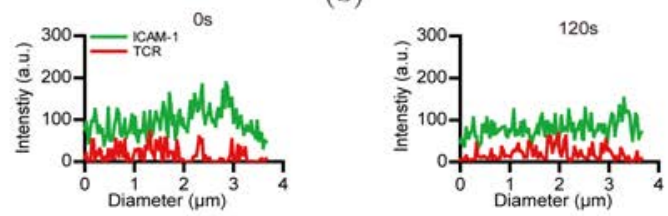

(c)

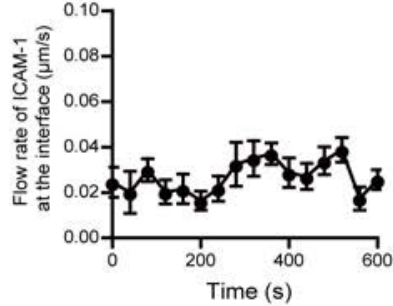

(e)

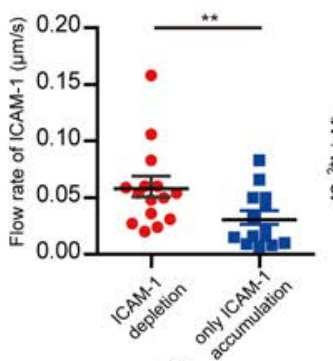

(f)

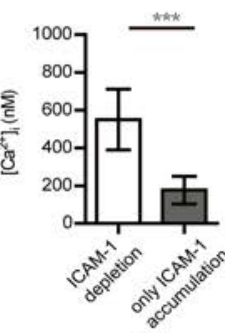

(g)

Fig. 2. The movement of ICAM-1 in the immature IS, which only accumulated ICAM-1 in the IS, and calcium release in a T-cell forming an immature synapse: (a) The relationship between the diameter change of the area of ICAM-1 depletion and calcium signaling in a T-cell. The movement of ICAM-1 at the interface of the synapse and calcium signaling in the T-cell contacted by the DC was detected by confocal microscopy with frames per $40 \mathrm{~s}$. (b) The imaging of calcium signaling in a T-cell and its contact with a DC. Bar $=2 \mu \mathrm{m}$. (c) Analysis of the distribution of the fluorescent values of ICAM-1 and TCR along the diameter of the synapse at $0 \mathrm{~s}$ and $120 \mathrm{~s}$. (d) Kymograph analysis of the relationship between ICAM-1 movement and calcium release in a T-cell. (e) The flow rate of ICAM-1 in the synapse during formation of T-DC contact. (f) The flow rate of ICAM-1 in the T-DC contact that had ICAM1 depletion in the center of the synapse or in the T-DC contact that only had ICAM-1 accumulation. (mean \pm s.e.m., $n=15$, three independent experiments), ${ }^{* *} p<0.01$ (two-tailed Student's $t$-test). (g) The concentration of calcium in T-cells which formed an IS with ICAM-1 depletion or that in T-cells which formed an IS with only ICAM-1 accumulation (mean \pm s.e.m., $n=25$, three independent experiments), ${ }^{* * *} p<0.001$ (two-tailed Student's $t$-test).

not find a trend for the movement of ICAM-1 from the center to the periphery of the synapse (Fig. 2(d)). The flow rate of ICAM-1 in the IS with only ICAM-1 accumulation fluctuated in the range of $0.02-0.06 \mu \mathrm{m} / \mathrm{s}$ (Fig. 2(e)). The average flow rate of ICAM-1 in the IS with only ICAM-1 accumulation was lower than that in the IS that had ICAM-1 depletion at the center of the synapse (Fig. 2(f), $\left.n=15,{ }^{* *} p<0.01\right)$. The calcium signaling in $\mathrm{T}$ cells forming mature ISs, which had ICAM-1 depletion at the center of the synapse, was higher than that in T-cells forming immature ISs, which only had ICAM-1 accumulation at the interface of T-DC (Fig. 2(g)).

Both the bullseye ISs and the multifocal ISs were mature ISs and had ICAM-1 depletion at the center of the synapse. The diameter of the ICAM-1 depletion area in the multifocal IS was larger than that in the bullseye IS (Supplementary Fig. 2(f)). Moreover, the calcium concentration in T-cells that formed a multifocal IS was higher than that formed a bullseye IS (Supplementary Fig. 2(g)). All of these results indicated that ICAM-1 depletion in the synapse might correlate with sustained calcium release in T-cells, while the movement of ICAM-1 in IS might affect calcium release in T-cells.

\subsection{ICAM-1 depletion at the center of the IS occurred only at the interface of antigen-specific T-cell-DC}

In our experiment, we analyzed the synapses formation between CD4+ T-cells from OT-II 
transgenic mice and DCs pulsed OVA antigen, and the synapses formation between CD4+ T-cells which include SEB antigen-specific T-cells and nonspecific T-cells from wild type mice, and DCs pulsed SEB antigen. For OVA antigen stimulation, ICAM1 depletion at the center of synapses occurred at $81.6 \pm 4.4 \%$ of T-DC contact, while $10 \pm 1.15 \%$ of T-DC contact failed to deplete ICAM-1 and formed immature synapses with ICAM-1 only accumulating at the interface of T-DC contact. Additionally, $8.3 \pm 3.75 \%$ of T-cells failed to form synapses with DCs. However, for SEB stimulation, the percentages of ISs with ICAM-1 depletion and ISs without ICAM-1 depletion were $60 \pm 7.6 \%$ and $18.3 \pm 6.0 \%$, respectively. In our model, the ICAM-1-GFP-DC2.4 cell line was a stable, monoclonal screened, and transfected by ICAM-1-GFP. Thus, the difference in T-cell activation may be caused by factors from T-cells. We first considered whether antigen-specific T-cells were involved in differences in T-cell activation caused by ICAM-1 depletion.

To further investigate the factors that affect the mobility of ICAM-1, we imaged the IS formation between DCs and co-cultured antigen-specific T-cells or nonantigen specific T-cells, respectively. We found out that ICAM-1 depletion at the center of the IS occurred mostly at the interface of antigenspecific T-cells and DCs pulsed with OVA or SEB. The en face images revealed that bullseye ISs consisted of ICAM-1 molecules surrounding a unique TCR cluster, while multifocal ISs consisted of ICAM-1 clusters intermingling with many TCR clusters (Fig. 3(a)). However, nonantigen specific Tcells either had ICAM-1 accumulated only at the interface of T-cells and DCs or had no significant ICAM-1 accumulation (Fig. 3(b)). The distribution of TCR and ICAM-1 at both types of T-DC contacts was essentially diffused (Fig. 3(b)). Without antigen stimulation, $13.3 \pm 4.4 \%$ of T-cells formed contacts with DCs to only accumulate ICAM-1 at the interface of T-DC (Fig. 3(c)). The percentage of ICAM-1 depletion at the center of the synapses was higher than the percentage of only ICAM-1 accumulation in ISs in antigen-specific T-cells (Fig. 3(c), $\left.{ }^{* * *} p<0.001\right)$. Moreover, the percentage of ICAM-1 depletion at the center of synapses that formed between antigen-specific T-cells and DCs was greater than that formed between nonantigen-specific T-cells and DCs as well as than that formed in the T-DC contact without antigen stimulation (Fig. 3(c), $\left.{ }^{* * *} p<0.001\right)$. These results suggested that antigen-specific T-cells might prefer to form mature ISs with ICAM-1 depletion from the center of synapses, while the formation of mature ISs might correlate with the extension of ICAM-1 from the center to the periphery of synapses. Furthermore, the study showed that the level of calcium release in antigen-specific T-cells was higher than that in nonantigen-specific T-cells or T-cells without antigen stimulation (Fig. $3(\mathrm{~d}),{ }^{* * *} p<0.001$ ). Thus, the depletion of ICAM- 1 at the synapse may preferentially occur in antigen-specific T-cells, which could induce a high level and sustained calcium release.

\subsection{Blocking ICAM-1 led to a decrease in the percentage of IS formation and a low level of calcium in T-cells}

To further analyze the role of ICAM-1 depletion in T-cell activation, mature DCs were pretreated with anti-ICAM-1 antibody to block ICAM-1, then pulsed with antigen and co-cultured with $\mathrm{T}$ cells. With anti-ICAM-1 antibody treatment, $\mathrm{T}$ cells failed to make effective contacts with DCs, while the percentage of T-DC contact decreased (Figs. 4(a) and 4(b)). The percentages of ICAM-1 accumulation and depletion in ISs decreased significantly (Fig. 4(b)). With anti-ICAM-1 antibody treatment, the flow rate of ICAM-1 in ISs decreased (Fig. 4(c)), while the level of calcium in T-cells was lower than that in T-cells without treatment. The peak of calcium signaling in T-cells that made contact with anti-ICAM-1 antibody treated DCs was lower than that in T-cells without treatment (Fig. 4(d)). Furthermore, these results were replicated by down-regulation of ICAM-1 expression with siRNA-ICAM-1 in DCs (Supplementary Fig. 1(c)). Therefore, the movement of ICAM-1 is important for calcium release in T-cells during IS formation.

\subsection{ICAM-1 depletion at the center of synapses was correlated with the movement of $L F A-1$}

LFA-1 is the ligand of ICAM-1 and a co-stimulated factor for T-cell activation. To investigate how the mobility of ICAM-1 influenced calcium release in Tcells, we labeled T-cells with anti-LFA-1 antibody 
and co-cultured them with ICAM-1-EGFP/DC2.4 cells. ICAM-1 co-localized with accumulated LFA-1 in both types of mature ISs (Fig. 5(a) and Supplementary Fig. 3(a)). By imaging the dynamic process of T-cells contacting DCs, we found at early time points (from $0 \mathrm{~s}$ to $300 \mathrm{~s}$ ) LFA-1 only co-localized with the most central region of accumulated ICAM-1 (Fig. 5(a) and Supplementary Fig. 3(a)). After incubation for $300 \mathrm{~s}$, LFA-1 molecules co-localized with the entire ICAM-1-rich region of ISs. Kymograph analysis showed the depletion of ICAM-1 at the center of the synapse occurred before
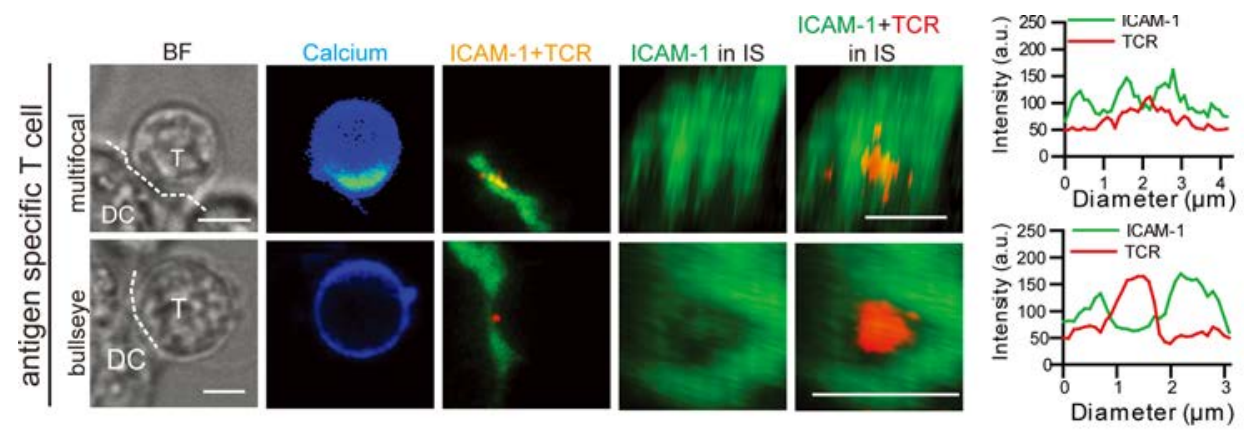

(a)
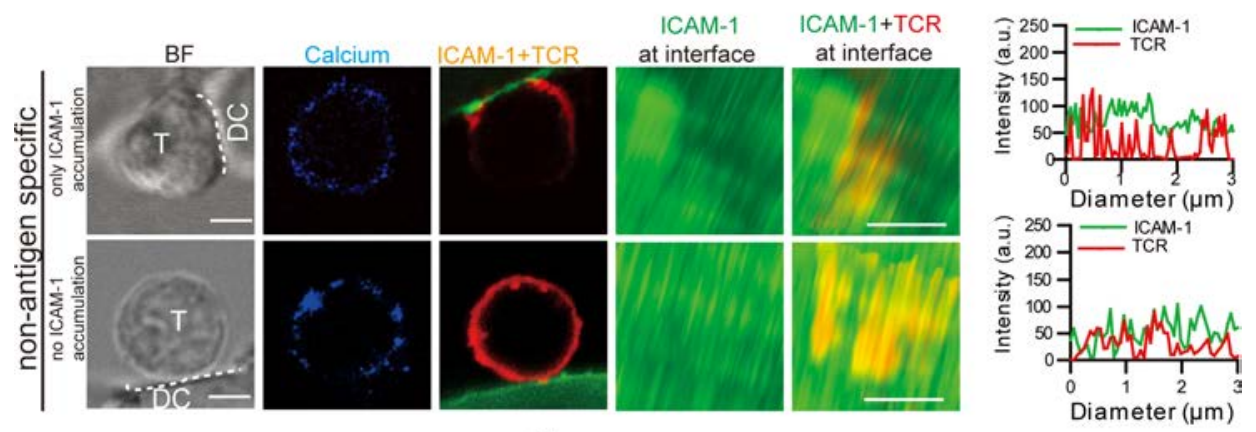

(b)

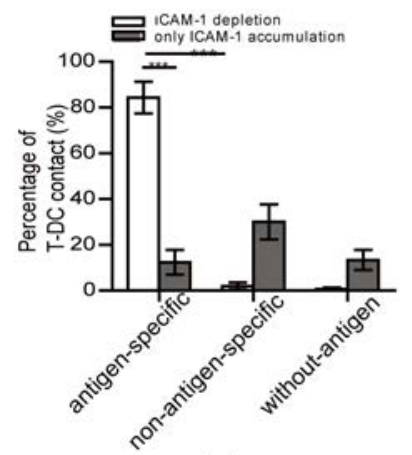

(c)

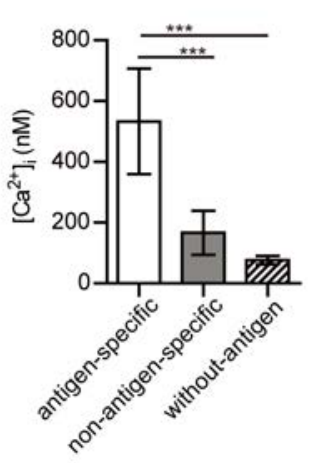

(d)

Fig. 3. The localization of ICAM-1 in the IS formed between an antigen-specific/nonspecific T-cell and a DC: (a) The types of synapses formed between an antigen-specific T-cell and a DC. A multifocal IS that had multiple sites for ICAM-1 depletion (the left and upper line) and a bullseye IS that had ICAM-1 depletion in the center of the IS (the left and down line) were formed. The fluorescent distribution of ICAM-1 and TCR along the diameter of the two types of synapses was shown on the right panel. Bar $=2 \mu \mathrm{m}$. (b) The synapses formed between a nonspecific antigen T-cell and a DC. ISs with only ICAM-1 accumulated at the contact of T-DC or without ICAM-1 accumulation at the contact of T-DC were shown. Bar $=2 \mu \mathrm{m}$. (c) Percentage of T-DC contact which had ICAM-1 depletion or only ICAM-1 accumulation at antigen-specific-T-cell-DC contact, nonspecific- T-cell-DC contact, or T-DC contact without antigen. (d) The calcium response in antigen-specific T-cells with ICAM-1 depletion at T-DC contact, nonspecific T-cells with only ICAM-1 accumulation at T-DC contact and T-cells without antigens at contact DC. (mean \pm s.e.m., $n=25$, three independent experiments), ${ }^{* * *} p<0.001$ (two-tailed Student's $t$-test). 


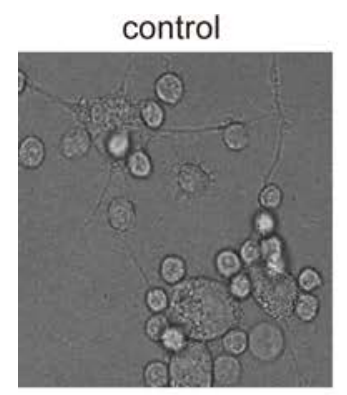

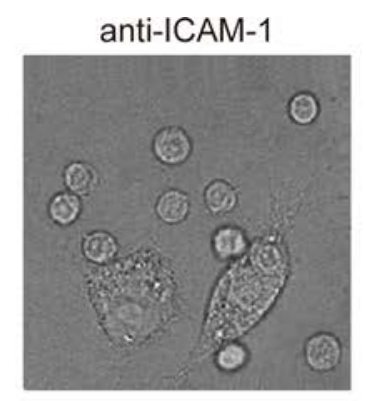

(a)

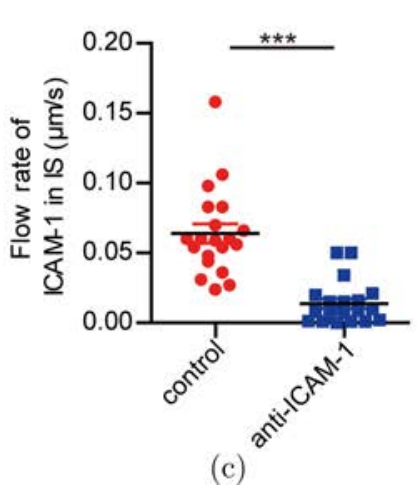

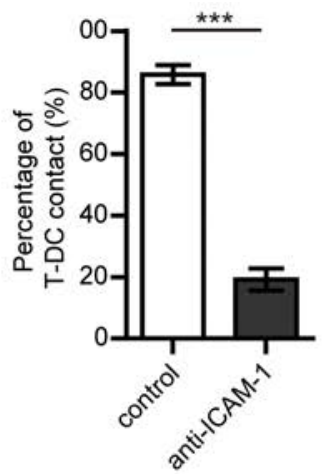
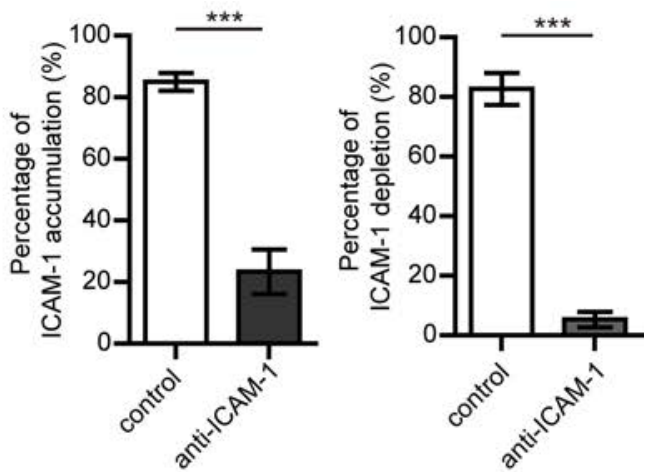

(b)

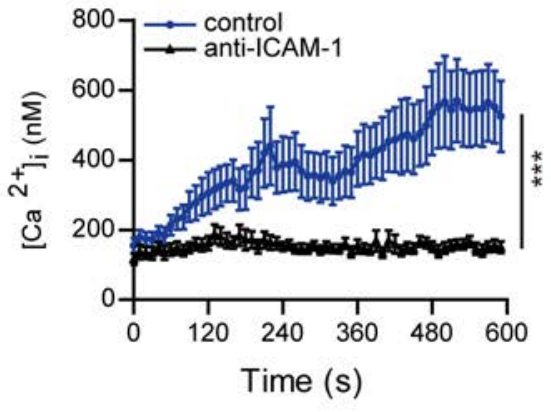

(d)

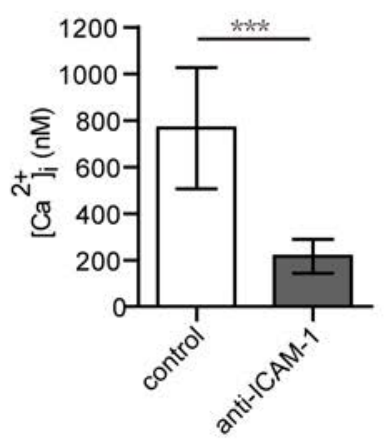

Fig. 4. Blocking ICAM-1 inhibited the accumulation of ICAM-1 in ISs and calcium release in T-cells: (a) With/without anti-ICAM-1 antibody treatment, the contact of T-DC was imaged, Bar $=10 \mu \mathrm{m}$. (b) With/without anti-ICAM-1 antibody treatment, the percentages of T-DC contact, ICAM-1 accumulation in the IS and ICAM-1 depletion in the IS. (c) The flow rate of ICAM-1 in the synapses with/without anti-ICAM-1 antibody treatment. (mean \pm s.e.m., $n=20$, three independent experiments), ${ }^{* * *} p<0.001$ (two-tailed Student's $t$-test). (d) With/without anti-ICAM-1 antibody treatment, the calcium release in $\mathrm{T}$ cells contacting DCs was measured. (mean \pm s.e.m., $n=20$, three independent experiments), ${ }^{* * *} p<0.001$ (two-tailed Student's $t$-test).

the LFA-1 depletion at the synapse (Fig. 5(b), as the white arrow shows). The extension of LFA-1 from the center to the periphery might follow the mobility of ICAM-1 (Fig. 5(b)). However, for the synapse with only ICAM-1 accumulation, LFA-1 accumulated and co-localized with ICAM-1 at the contact of T-DC, and lost its ability to move from the center to the periphery at synapses with only ICAM-1 accumulation (Figs. 5(c) and 5(d)).

By blocking LFA-1, the percentage of T-DC contacts decreased (Figs. 5(e) and 5(f)). The T-DC contacts failed to accumulate ICAM-1 at the interface and lost the ability to deplete ICAM-1 at the center of the IS. LFA-1 could not accumulate at the interface of T-DC (Fig. 5(e)). In addition, by blocking ICAM-1, LFA-1 failed to accumulate into the synapse (Fig. 5(e)). These results indicated that the binding of ICAM-1-LFA-1 was a factor affecting the depletion of ICAM-1 at the center of ISs.

\subsection{The movement of $L F A-1$ affected calcium release in $T$-cells by controlling the location of ORAI1 and mitochondria at the IS}

With anti-LFA-1 antibody pretreatment, both the flow rate of LFA-1 and the calcium release in T-cells decreased (Fig. 5(g)). These results indicated that LFA-1 might affect calcium release in T-cells. To analyze how LFA-1 affected calcium release in Tcells, we imaged the location of LFA-1, ORAI1 and mitochondria at the synapse. ORAI1 is a key member of the calcium release-activated calcium channel protein family in $\mathrm{CD} 4^{+} \mathrm{T}$-cells and plays an important role in increasing the local calcium concentration in T-cells. ${ }^{17}$ Mitochondria are essential for calcium homeostasis in fundamental cellular functions such as metabolism and signaling in a large variety of cell types. ${ }^{25}$ Interestingly, the ability of mitochondria to regulate calcium signaling 


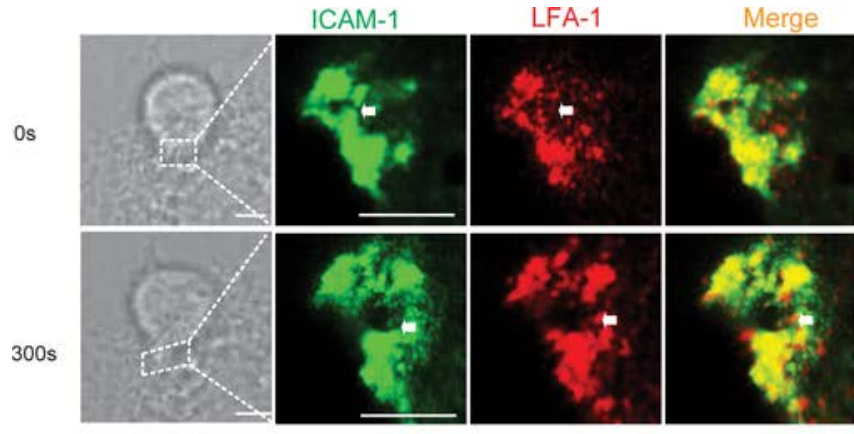

(a)

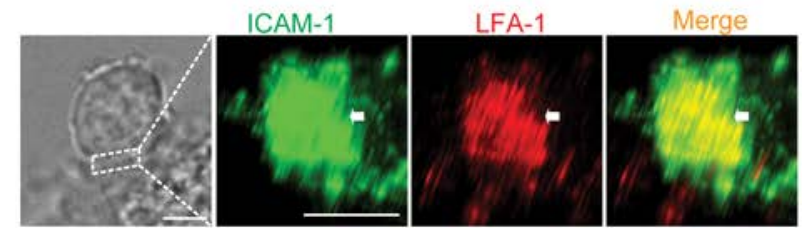

(c)

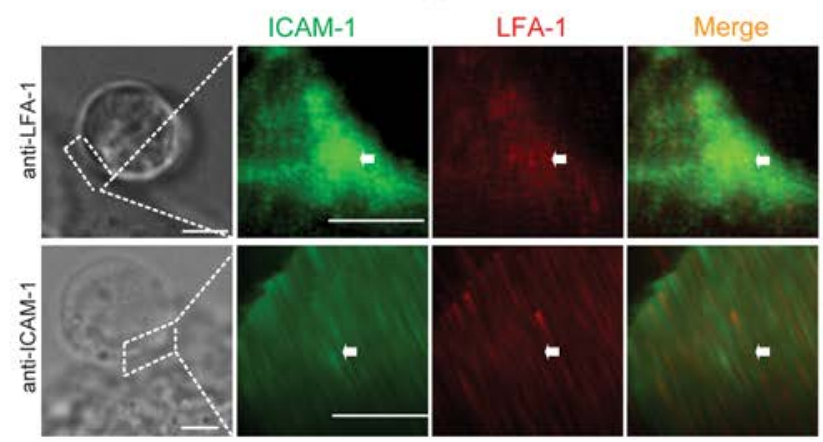

(e)

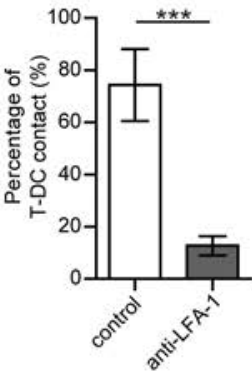

(f)
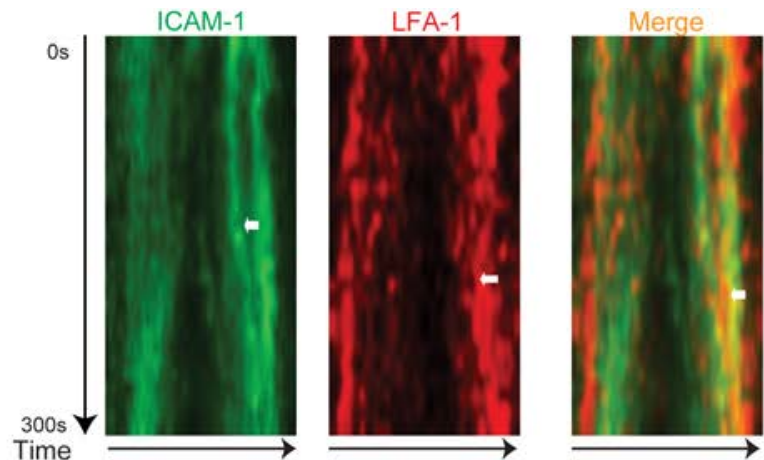

Diameter of IS $(\mu \mathrm{m})$

(b)

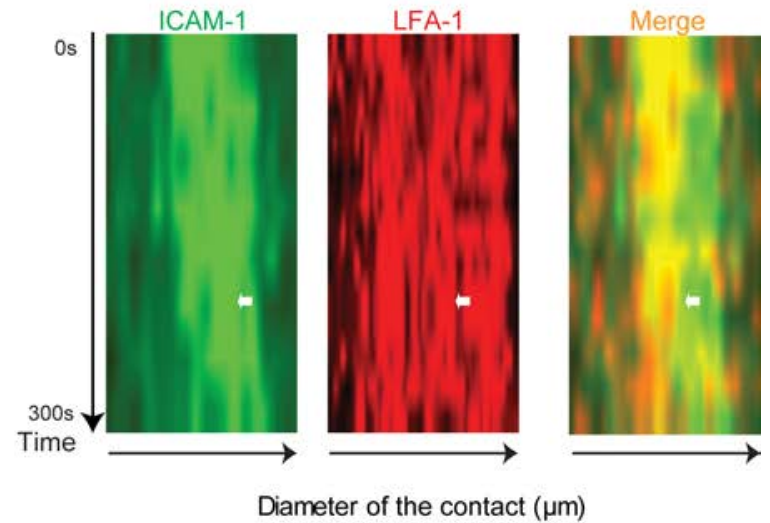

(d)

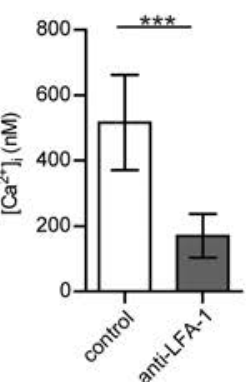

(g)

Fig. 5. The movement of ICAM-1 and LFA-1 in ISs: (a) The localization of ICAM-1 and LFA-1 at a bullseye IS that had ICAM-1 depletion in the center of the IS. It was measured at $0 \mathrm{~s}$ and $300 \mathrm{~s}$ after a T-cell contacted a DC. Bar $=2 \mu \mathrm{m}$. (b) Kymograph analysis of the dynamic change of ICAM-1 and LFA-1 along the diameter of the synapse from $0 \mathrm{~s}$ to $300 \mathrm{~s}$. The white arrows in the panel indicate the trend of ICAM-1 and LFA-1 movement from the center to the periphery of the IS. (c) The localization of ICAM-1 and LFA-1 at T-DC contact with only ICAM-1 accumulation. (d) Kymograph analysis of the dynamic change of ICAM-1 and LFA-1 along the diameter of the synapse that only had ICAM-1 accumulation in the T-DC contact. (e) With treatment of anti-ICAM-1 antibody and anti-LFA-1 antibody, the localization of ICAM-1 and LFA-1 were measured at T-DC contact. (f) With/without treatment of anti-LFA-1 antibody, the percentage of T-DC contact was measured. (g) With/without treatment of anti-LFA-1 antibody, the concentration of calcium in the T-cell was measured. The arrows in figures (b) and (d) point the movement of direction of accumulated ICAM-1 or LFA-1. The arrows in the figure (a) showed the center of synapse. The arrows in the figures (c) and (e) showed the interface of T- DC contact. 

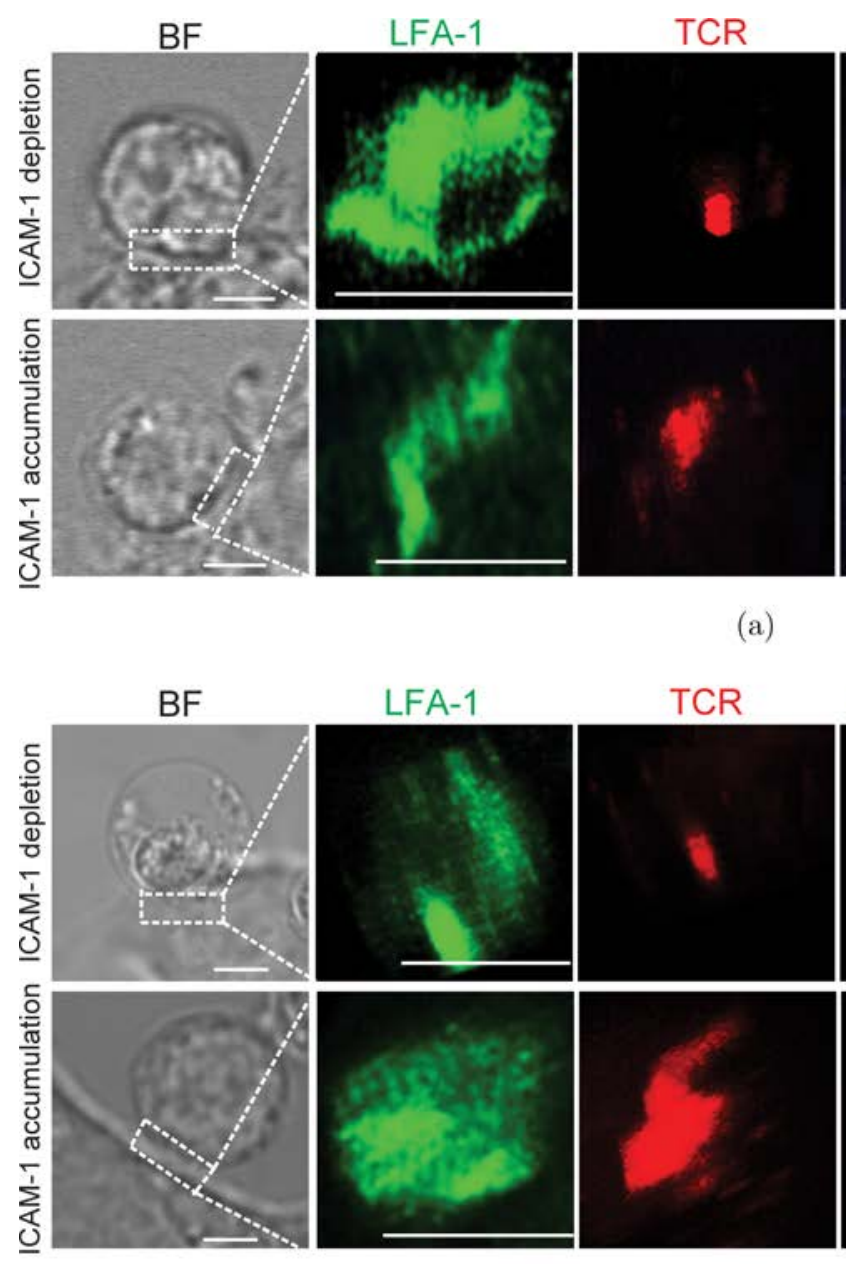

(c)

(a)
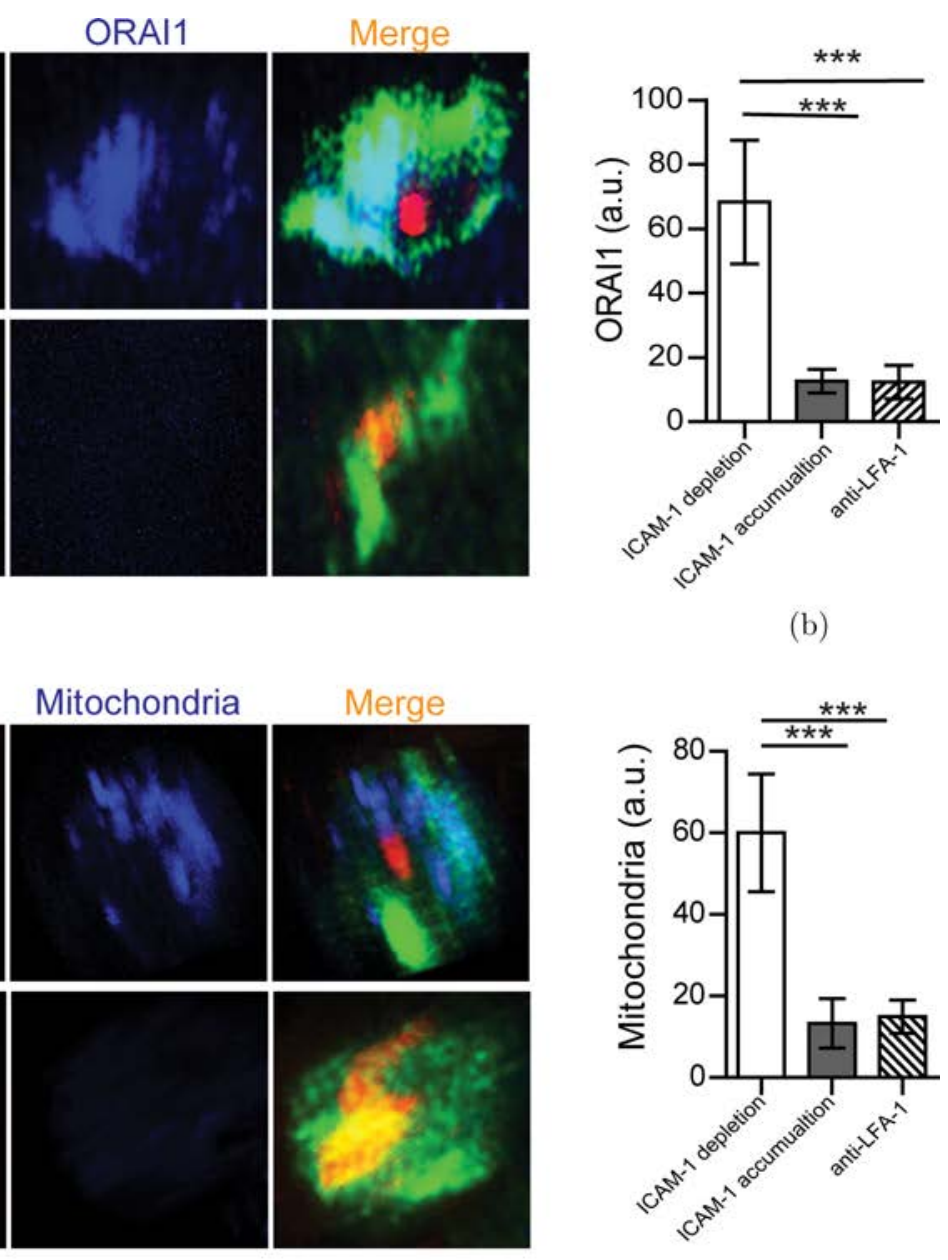

(d)

Fig. 6. The co-localization of ORAI1, mitochondria and LFA-1 in mature ISs with ICAM-1 depletion or immature ISs with only ICAM-1 accumulation: (a) The localization of ORAI1 and LFA-1 in a mature IS (bullseye IS) and an immature IS. Bar $=2 \mu$ m. (b) The fluorescence of ORAI1 in the mature IS, immature IS, and IS with anti-LFA-1 antibody treatment. (mean \pm s.e.m., $n=25$, three independent experiments), ${ }^{* * *} p<0.001$ (two-tailed Student's $t$-test). (c) The localization of mitochondria and LFA-1 in a type of mature IS (bullseye IS) with ICAM-1 depletion or immature IS with only ICAM-1 accumulation. (d) The fluorescence of mitochondria in the mature IS, immature IS and IS with anti-LFA-1 antibody treatment. (mean \pm s.e.m., $n=25$, three independent experiments), ${ }^{* * *} p<0.001$ (two-tailed Student's $t$-test).

depends on their sub-cellular localization. ${ }^{26}$ In our experiment, we found out that ORAI1 could colocalize with LFA-1 in mature ISs that had ICAM-1 depletion, while it could not co-localize with synapses with only ICAM-1 accumulation (Figs. 6(a) and 6(b) and Supplementary Fig. 4(a)). Mitochondria were accumulated into mature ISs and partly co-localized with LFA-1, while it could not accumulate into synapses with only ICAM-1 accumulation (Figs. 6(c) and 6(d) and Supplementary Fig. 4(b)). By blocking LFA-1 with anti-LFA-1 antibody, the fluorescent values of ORAI1 and mitochondria were greatly reduced (Figs. 6(b) and $6(d))$. LFA-1 failed to deplete at the center of the synapses, while ORAI1 and mitochondria failed to localize into the synapse (data not shown). These data suggested that LFA-1 could modulate the localization of ORAI1 and mitochondria in ISs.

\section{Discussion}

T-cell activation is an essential step for immune surveillance and immune response. Calcium signaling in T-cells is an initial marker of T-cell activation and is linked to cell functions. ${ }^{5,6,27}$ Moreover, the calcium signal in the activated T-cells must reach a cytosolic concentration ten-fold higher than that in the resting T-cells and be sustained for at least $2 \mathrm{~h}$ 
to trigger effective genes expression. ${ }^{7,28,29}$ For this purpose, many T-cell signaling molecules and calcium microdomains accumulate into ISs to sustain calcium signaling. ${ }^{7,17}$ However, whether the mobility of those molecules could affect the calcium signaling was unclear. Herein, by imaging live cells, we found out that the mobility of ICAM-1 in synapses was correlated with the initial calcium release in T-cells. In the process of IS formation, the accumulation and consequent depletion of ICAM-1 occurred, followed by calcium release and TCR accumulation into the center of the IS (Fig. 1). Without ICAM-1 accumulation or ICAM-1 depletion at the center of the IS or by blocking the movement of ICAM-1, the sustained and high level of calcium level could not be induced. Those results indicated ICAM-1 accumulation and depletion at the center of the IS was correlated with initial calcium release in T-cells (Supplementary Fig. 5). The movement ICAM-1 might trigger the initial calcium release in T-cells. In addition, ICAM-1 depletion at the center of the IS might provide a site for TCR to accumulate and localize at the center of the IS to recognize p-MHC, which might contribute to the sustained calcium level and T-cell activation. Whether TCR accumulation affects ICAM-1 movement or the initial calcium release affects TCR or ICAM-1 movement, needs further study.

By studying the mechanism for how ICAM-1 depletion induced calcium release in T-cells, we found out that the mobility of ICAM-1 was associated with the movement of LFA-1. The extension of ICAM-1 from the center to the periphery of synapses was accompanied with the mobility of LFA-1. Moreover, the movement of LFA-1 might influence the locations of ORAI1 and mitochondria in the IS, which were believed to regulate the localization of calcium influx in T-cells. This result is similar to the study of Contento $\mathrm{R}^{30}$ about the ICAM-1-LFA-1 binding results in mitochondria translocation to the IS and signaling in T-cell. We further proved that the depletion of ICAM-1 in the synapse is an important factor for calcium release in T-cells. The mobility of ICAM-1-LFA-1 bond clusters at sites of synapses might regulate calcium signaling in T-cells by controlling the localization of ORAI1 and mitochondria at the synapse (Supplementary Fig. 5).

Another possible mechanism of ICAM-1 depletion leading to calcium release is that ICAM-1 depletion at the center of synapses might be preparing an area for TCRs to localize at the center to form stable TCR-MHC-peptide contact, which is better for TCR signal pathway activation. The activation of the TCR signal pathway is believed to be essential for intracellular calcium release. TCR engagement with peptides conjugated to MHC on APCs leads to activation of signal transduction pathways, which promote a rapid release of calcium from the endoplasmic reticulum (ER) calcium stores. ${ }^{31-33}$

To analyze why ICAM-1 could accumulate and then be depleted at the center of synapses, we compared the ICAM-1 accumulation at the antigenspecific T-DC contact and nonspecific T-DC contact. We found out that antigen-specific T-cells preferred to form the mature IS that involved ICAM-1 accumulation and depletion. Nonantigenspecific T-cells formed immature ISs without ICAM-1 depletion. Thus, ICAM-1 depletion might be a characteristic to distinguish mature ISs from immature ISs and a defining characteristic for the contact of antigen-specific T-cells to DCs. For antigen-specific and nonspecific T-cells, the status of DCs is the same. For antigen-specific T-cells, antigen-specific TCRs recognize the antigen and cluster into many micro-TCR clusters, which keep moving on the surface of T-DC. However, for nonantigenspecific T-cells, TCRs cannot recognize antigens and fail to accumulate into the IS. TCRs recognize antigens and bind MHCs to activate cytoskeletal proteins, such as F-actin, by the MHC signaling pathway. F-actin may regulate the mobility of ICAM-1. ${ }^{13}$ Thus, the movement of antigen-specific TCR might be the reason for ICAM-1 accumulation and depletion in the formation of mature ISs. The movement of micro-TCR clusters at the interface of T-DC might activate the F-actin in DCs by MHCs to promote ICAM-1 movement. However, those hypotheses need further study. In addition, antigenspecific T-cells are usually believed to be activated more efficiently than nonspecific T-cells to induce the antigen-specific immune surveillance. However, molecular mechanism for how antigen-specific $\mathrm{T}$ cells are activated in a short time, while nonspecific T-cells are not, is still unclear. ICAM-1 accumulation and depletion at the IS to induce the initial calcium response in T-cells and form a mature IS to supply T-cell activation might be a factor for effective activation in antigen-specific T-cells. The correlations and mechanisms of calcium release in nonspecific T-cells and the movement of ICAM-1 need further investigation. The immature synapse has been believed to be signaled at time scales of 
1-5 min $^{1}$ during which adhesion molecules ICAM-1LFA-1 bond with each other to signal the start of the synapse formation process. At the timescale mentioned, these larger bonds are substituted by smaller TCR-pMHC molecules and associated coreceptors to take over the signaling of immunological synapse. Recently, some studies questioned the minute-time scale and suggested that the immature synapse occurs at the time scale of seconds. ${ }^{34-36}$ Our study confirmed this finding by using the live-cell calcium imaging. The timescale switches from minutes to seconds might be influenced by the movement of ICAM-1, which correlates with calcium release.

\section{Acknowledgment}

This work was supported by the National Major Scientific Research Program of China (Grant No. 2011CB910404), the National Nature Science Foundation of China (Grant Nos. 61227017, 31400772 and 81273215) and the National Science Fund for Distinguished Young Scholars (Grant No. 61425006), the grants of the Project for Laureate of Taishan Scholar (Grant No. ts201511075) and the Innovation Project of Shandong Academy of Medical Sciences, the Projects of medical and health technology development program in Shandong province (No. 2015WS0194); the science and technology program from Shandong Academy of Medical Sciences (No. 2015-25).

\section{References}

1. A. Grakoui, S. K. Bromley, C. Sumen, M. M. Davis, A. S. Shaw, P. M. Allen, M. L. Dustin, "The immunological synapse: A molecular machine controlling T-cell activation," Science 285, 221-227 (1999).

2. C. R. Monks, B. A. Freiberg, H. Kupfer, N. Sciaky, A. Kupfer, "Three-dimensional segregation of supramolecular activation clusters in T cells," Nature 395, 82-86 (1998).

3. C. Brossard, V. Feuillet, A. Schmitt, C. Randriamampita, M. Romao, G. Raposo, A. Trautmann, "Multifocal structure of the T-cell - dendritic cell synapse," Eur. J. Immunol. 35, 1741-1753 (2005).

4. E. Hailman, W. R. Burack, A. S. Shaw, M. L. Dustin, P. M. Allen, "Immature CD4(+)CD8(+) thymocytes form a multifocal immunological synapse with sustained tyrosine phosphorylation,"
Immunity 16, 839-848 (2002).

5. N. Joseph, B. Reicher, M. Barda-Saad, "The calcium feedback loop and T-cell activation: How cytoskeleton networks control intracellular calcium flux," Biochim. Biophys. Acta. 1838, 557-568 (2014).

6. A. Babich, J. K. Burkhardt, "Coordinate control of cytoskeletal remodeling and calcium mobilization during T-cell activation," Immunol. Rev. 256, 80-94 (2013).

7. M. F. Krummel, M. D. Cahalan, "The immunological synapse: A dynamic platform for local signaling," J. Clin. Immunol. 30, 364-372 (2010).

8. J. Delon, N. Bercovici, G. Raposo, R. Liblau, A. Trautmann, "Antigen-dependent and -independent responses triggered in T cells by dendritic cells compared with B cells," J. Exp. Med. 188, 14731484 (1998).

9. K. H. Lee, A. R. Dinner, C. Tu, G. Campi, S. Raychaudhuri, R. Varma, T. N. Sims, W. R. Burack, H. Wu, J. Wang, O. Kanagawa, M. Markiewicz, P. M. Allen, M. L. Dustin, A. K. Chakraborty, A. S. Shaw, "The immunological synapse balances T-cell receptor signaling and degradation," Science 302, 1218-1222 (2003).

10. D. J. Irvine, M. A. Purbhoo, M. Krogsgaard, M. M. Davis, "Direct observation of ligand recognition by T cells," Nature 419, 845-849 (2002).

11. P. Revy, M. Sospedra, B. Barbour, A. Trautmann, "Functional antigen-independent synapses formed between T cells and dendritic cells," Nat. Immunol. 2, 925-931 (2001).

12. M. F. Krummel, M. M. Davis, "Dynamics of the immunological synapse: Finding, establishing and solidifying a connection," Curr. Opin. Immunol. 14, 66-74 (2002).

13. W. A. Comrie, S. Li, S. Boyle, J. K. Burkhardt, "The dendritic cell cytoskeleton promotes T-cell adhesion and activation by constraining ICAM-1 mobility," J. Cell. Biol. 208, 457-473 (2015).

14. Z. Fan, A. Marki, S. McArdle, Z. Mikulski, E. Gutierrez, B. Engelhardt, U. Deutsch, M. Ginsberg, A. Groisman, K. Ley, "Neutrophil recruitment limited by high affinity bent $\beta 2$ integrin binding ligand in cis," Nat. Commun. 7, 12658 (2016).

15. Z. Fan, K. Ley, "Leukocyte arrest: Biomechanics and molecular mechanisms of $\beta_{2}$ integrin activation," Biorheology 52, 353-77 (2015).

16. N. Dixit, I. Yamayoshi, A. Nazarian, S. I. Simon, "Migrational guidance of neutrophils is mechanotransduced via high-affinity LFA-1 and calcium flux," J. Immunol. 187, 472-481 (2011).

17. A. Quintana, M. Pasche, C. Junker, D. Al-Ansary, H. Rieger, C. Kummerow, L. Nunez, C. Villalobos, P. Meraner, U. Becherer, J. Rettig, B. A. Niemeyer, 
M. Hoth, "Calcium microdomains at the immunological synapse: How ORAI channels, mitochondria and calcium pumps generate local calcium signals for efficient T-cell activation," EMBO J 30, 38953912 (2011).

18. W. Lin, Z. Fan, Y. Suo, Y. Deng, M. Zhang, J. Wang, X. Wei, Y. Chu, "The bullseye synapse formed between CD4+ T-cell and staphylococcal enterotoxin B-pulsed dendritic cell is a suppressive synapse in T-cell response," Immunol. Cell Biol. 93, 99-110 (2015).

19. T. He, C. Tang, S. Xu, T. Moyana, J. Xiang, "Interferon gamma stimulates cellular maturation of dendritic cell line DC2.4 leading to induction of efficient cytotoxic T-cell responses and antitumor immunity," Cell Mol. Immunol. 4, 105-111 (2007).

20. Z. Shen, G. Reznikoff, G. Dranoff, K. L. Rock, "Cloned dendritic cells can present exogenous antigens on both MHC class I and class II molecules," $J$. Immunol. 158, 2723-2730 (1997).

21. S. Y. Tseng, J. C. Waite, M. Liu, S. Vardhana, M. L. Dustin, "T cell-dendritic cell immunological synapses contain TCR-dependent CD28-CD80 clusters that recruit protein kinase C theta," J. Immunol. 181, 4852-4863 (2008).

22. R. S. Friedman, P. Beemiller, C. M. Sorensen, J. Jacobelli, M. F. Krummel, "Real-time analysis of T-cell receptors in naive cells in vitro and in vivo reveals flexibility in synapse and signaling dynamics," J. Exp. Med. 207, 2733-2749 (2010).

23. W. Lin, Y. Suo, Y. Deng, Z. Fan, Y. Zheng, X. Wei, Y. Chu, "Morphological change of CD4(+) T-cell during contact with DC modulates T-cell activation by accumulation of F-actin in the immunology synapse," BMC Immunol. 16, 49 (2015).

24. A. Babich, S. Li, R. S. O'Connor, M. C. Milone, B. D. Freedman, J. K. Burkhardt, "F-actin polymerization and retrograde flow drive sustained PLCgamma1 signaling during T-cell activation," J. Cell Biol. 197, 775-787 (2012).

25. M. R. Duchen, "Mitochondria and calcium: From cell signalling to cell death," J. Physiol. 529 Pt 1, 57-68 (2000).
26. A. Quintana, C. Schwindling, A. S. Wenning, U. Becherer, J. Rettig, E. C. Schwarz, M. Hoth, "T cell activation requires mitochondrial translocation to the immunological synapse," Proc. Natl. Acad. Sci. USA. 104, 14418-14423 (2007).

27. W. Lin, Z. C. Fan, "Immunological Synapse Molecules", J. Immunobiol. 1, 1000111 (2016).

28. C. M. Fanger, M. Hoth, G. R. Crabtree, R. S. Lewis, "Characterization of T-cell mutants with defects in capacitative calcium entry: Genetic evidence for the physiological roles of CRAC channels," J. Cell Biol. 131, 655-667 (1995).

29. P. A. Negulescu, N. Shastri, M. D. Cahalan, "Intracellular calcium dependence of gene expression in single T lymphocytes," Proc. Natl. Acad. Sci. USA. 91, 2873-2877 (1994).

30. R. L. Contento, S. Campello, A. E. Trovato, E. Magrini, F. Anselmi, A. Viola, "Adhesion shapes $\mathrm{T}$ cells for prompt and sustained T-cell receptor signalling," EMBO J. 29, 4035-4047 (2010).

31. R. S. Lewis, "Calcium signaling mechanisms in T lymphocytes," Annu. Rev. Immunol. 19, 497-521 (2001).

32. L. E. Samelson, "Signal transduction mediated by the T-cell antigen receptor: The role of adapter proteins," Annu. Rev. Immunol. 20, 371-394 (2002).

33. S. Feske, "Calcium signalling in lymphocyte activation and disease," Nat. Rev. Immunol. 7, 690-702 (2007).

34. D. R. Bush, A. K. Chattopadhyay, "Contact time periods in immunological synapse," Phys. Rev. E Stat. Nonlin. Soft Matter Phys. 90, 042706-1042706-6 (2014).

35. D. R. Bush, A. K. Chattopadhyay, "Temporal dynamics in an immunological synapse: Role of thermal fluctuations in signaling," Phys. Rev. E Stat. Nonlin. Soft Matter Phys. 92, 012706 (2015).

36. A. K. Chattopadhyay, N. J. Burroughs, "Close contact fluctuations: The seeding of signalling domains in the immunological synapse," Europhys Lett. 77, 48003 (2007). 